\title{
Impact of Filter Characteristics and Optical Signal Parameters on Interchannel Crosstalk
}

\author{
Agnieszka Kowalczyk and Krzysztof Perlicki
}

\begin{abstract}
In this paper we present analysis of the influence of filter characteristics of Wavelength Division Multiplexing network components and transmission parameters over interchannel crosstalk for symmetric and asymmetric transmission. The simulation was carried out for bit rate $10 \mathrm{~Gb} / \mathrm{s}, 40 \mathrm{~Gb} / \mathrm{s}$ and $100 \mathrm{~Gb} / \mathrm{s}$ considering the following modulations: DPSK, DQPSK and OOK. The obtained results show that OOK and DQPSK modulation are better than DPSK modulation in terms of crosstalk occurring during the transmission of signals in Wavelength Division Multiplexing systems.
\end{abstract}

Keywords-fiber optics transmsission, Wavelength Division Multiplexing, optical filter, interchannel crosstalk

\section{INTRODUCTION}

$\mathbf{O}$ PTICAL TRANSPORT CAPACITIES have been growing significantly in the last few years following the explosive demand for high bandwidth Internet services. The data transmission rates are now moving from $10 \mathrm{~Gb} / \mathrm{s}$ to $40 \mathrm{~Gb} / \mathrm{s}$ and $100 \mathrm{~Gb} / \mathrm{s}$ on a wavelength (optical channel) and wavelengths are more densely spaced. The optical WDM (Wavelength Division Multiplexing) transmission systems is evolving from closed systems to open systems, where the optical layer is designed in a way that allows new transmitters and receivers to be connected to it without requiring changes to the physical layer not to the planning and management tools that are needed to operate it. [1], [2]. Nowdays, the single WDM transmission system must be able to transmitted different optical signal modulation formats and different bit rates [3], [4]. One of the most important issues for the design of the current and future WDM systems is relationship between transmitted signal modulation format, bit rate, optical channel spacing and parameters of multiplexer and demultiplexer filter [5]. In this paper, we present a numerical simulation of the impact of transmitted optical signal parameters and parameters of multiplexer and demultiplexer filter on WDM channels crosstalk. The simulation setup is focused on optical transmission system with $10 \mathrm{~Gb} / \mathrm{s}, 40 \mathrm{~Gb} / \mathrm{s}$ or $100 \mathrm{~Gb} / \mathrm{s}$ optical channels. The following signal modulation formats are taken into account: DPSK (Differential Phase Shift Keying), DQPSK (Differential Quadrature Phase Shift Keying) and OOK (Onoff Keying) [6].

A. Kowalczyk and K. Perlicki are with the Institute of Telecommunications, Warsaw University of Technology, Nowowiejska 15/19, 00-665 Warsaw, Poland (e-mail: perlicki@tele.pw.edu.pl).

\section{NUMERICAL SimUlations}

The simulation setup is shown in Fig. 1. The simulated setup consists of: 4 transmitters, multiplexer $4 \times 1$, demultiplexer $1 \times 4$ and fiber optics link SSMF (Standard Single Mode Fiber). In the system setup 4 channels were used, which were separated with $50 \mathrm{GHz}$ channel spacing. The channel frequencies were selected according to ITU-T G.694.1 recommendation $(193.05 \mathrm{THz}, 193.10 \mathrm{THz}, 193.15 \mathrm{THz}, 193.20$ $\mathrm{THz}$ [7]. The $3 \mathrm{~dB}$ bandwidth of the multiplexer and demultiplexer filter varied between $20 \mathrm{GHz}$ and $60 \mathrm{GHz}$ for all tested modulations and bit rates. There were considered two shapes of filter transfer function: gaussian and rectangular. For gaussian filter the filter order changed from $1^{\text {st }}$ to $6^{\text {th }}$ order. In case of a rectangular filter stopband attenuation was changed from 20 to $70 \mathrm{~dB}$. Default filter had gaussian characteristics of second order. Due to the research the effects of transmission parameters and filters used in the system over interchannel crosstalk, nonlinear effects such as stimulated Raman scattering, stimulated Brillouin scattering, self-phase and cross-phase modulation and four wave mixing have been omitted. Additionally, optical loss, chromatic and polarization dispersion have been also neglected. Optical crosstalk was calculated for the Port No. 2 (Fig. 1). The simulation was carried out for bit rate $10 \mathrm{~Gb} / \mathrm{s}, 40 \mathrm{~Gb} / \mathrm{s}$ and $100 \mathrm{Gbt} / \mathrm{s}$ considering the following modulations: DPSK, DQPSK and OOK. Two cases were considered as well:

Case 1: Transmitted signals had the same bit rate for each channel.

Case 2: In the channel, which was considered for calculating crosstalk, the bit rate was $10 \mathrm{~Gb} / \mathrm{s}$, while in the adjacent channels signals were transmitted with a higher bit rate: $40 \mathrm{~Gb} / \mathrm{s}$ or $100 \mathrm{~Gb} / \mathrm{s}$.

Numerical simulations were realized with VPItranmission Maker (VPIsystems Inc.)

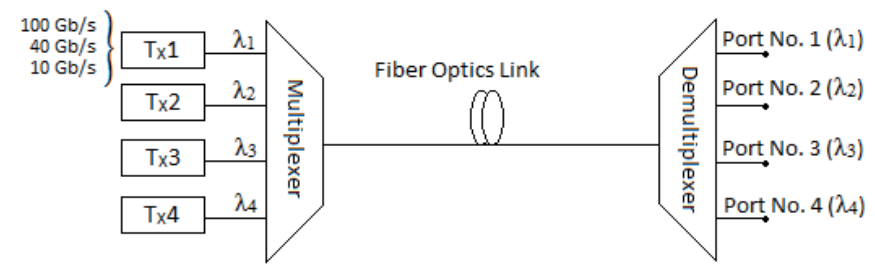

Fig. 1. Diagram of simulated DWDM system RX1, RX2 - receivers Schematics of simulated system; Tx1, Tx2, Tx3, Tx4 are transmitters of each wavelenght: $\lambda_{1}, \lambda_{2}, \lambda_{3}, \lambda_{4}$. Channel at the Port no.2 $\left(\lambda_{2}\right)$ was considered for calculating interchannel crosstalk. 


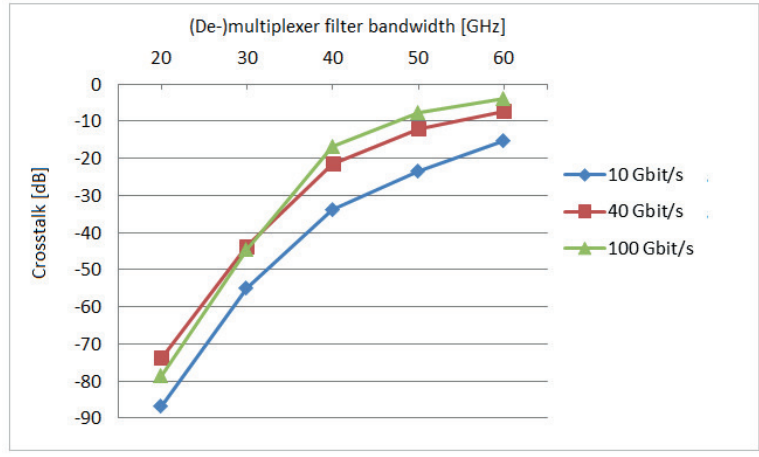

Fig. 2. Crosstalk values versus demultiplexer and multiplexer filter bandwidth for DPSK modulation, second order gaussian filter and different bit rates.

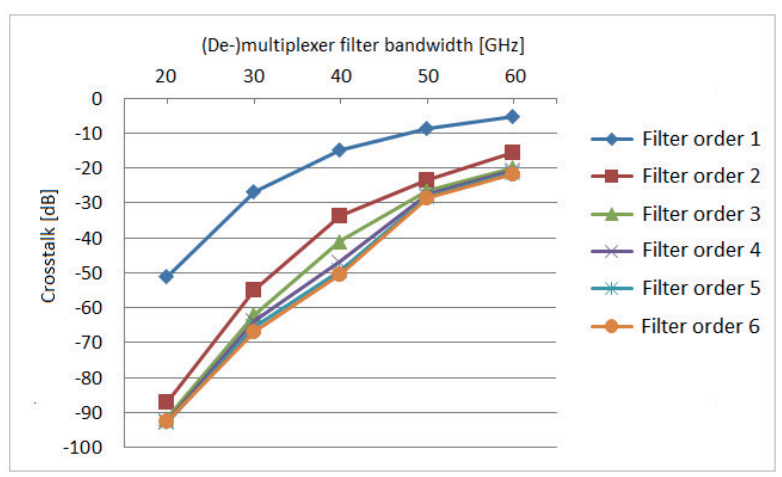

Fig. 3. Crosstalk values versus demultiplexer and multiplexer filter bandwidth for DPSK modulation, $10 \mathrm{~Gb} / \mathrm{s}$ bit rate and different gaussian filter order.

\section{NumericAl Results}

Figs. 2-15 show the results for case 1 (symmetric transmission). Here, transmitted signals had the same bit rate for each channel.

Simulations were performed for the WDM system shown in Fig. 1.

From Figs. 2, 6, 10 it can be concluded that level of crosstalk depends largely on bit rate in channel. This is because of the fact that for higher bit rate spectrum becomes wider. Obtaining $10 \mathrm{Gbit} / \mathrm{s}$ required filter bandwidth of approximately $55 \mathrm{GHz}$

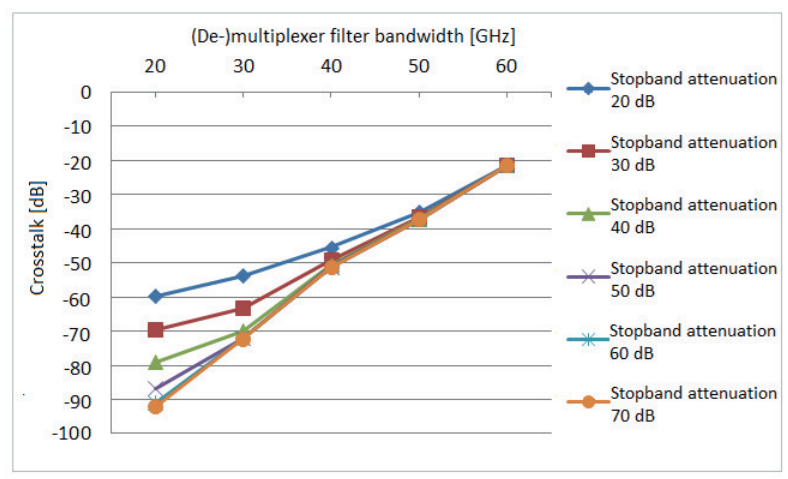

Fig. 4. Crosstalk values versus demultiplexer and multiplexer filter bandwidth for DPSK modulation, $10 \mathrm{~Gb} / \mathrm{s}$ bit rate and different stopband attenuation of rectangular filter characteristics.

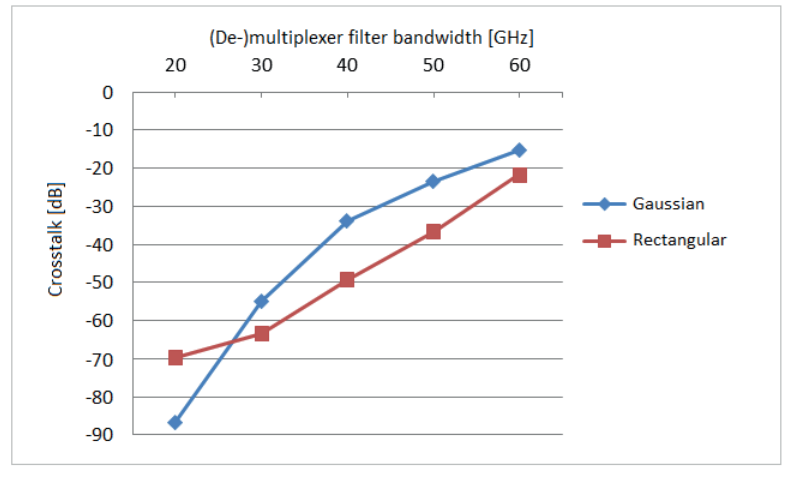

Fig. 5. Crosstalk values versus demultiplexer and multiplexer filter bandwidth for DPSK modulation, $10 \mathrm{~Gb} / \mathrm{s}$ bit rate and different types of filters: second order gaussian filter and rectangular filter with stopband attenuation $30 \mathrm{~dB}$.

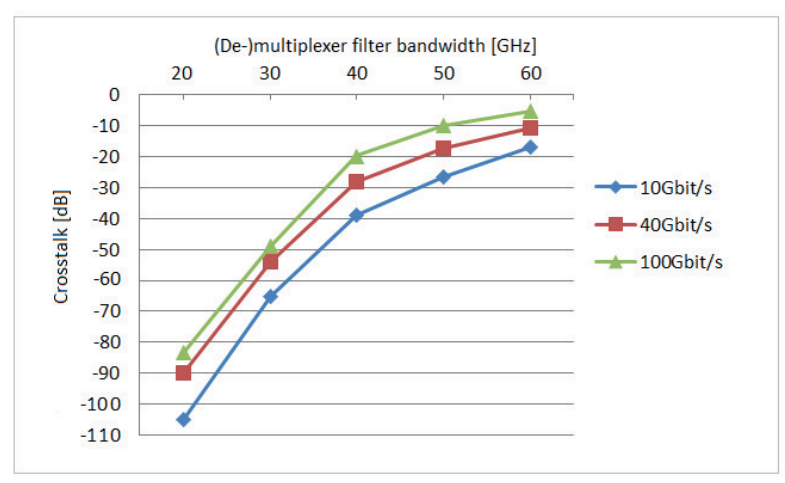

Fig. 6. Crosstalk values versus demultiplexer and multiplexer filter bandwidth for DQPSK modulation, second order gaussian filter and different bit rates.

for all tested modulation. For $40 \mathrm{Gbit} / \mathrm{s}$ it was necessary to use narrower filter bandwidth. In case of DPSK modulation and bit rate $100 \mathrm{Gbit} / \mathrm{s}$ acceptable level of crosstalk was achieved for around $40 \mathrm{GHz}$ of filter bandwidth. For $50 \mathrm{GHz}$ crosstalk amounted $-8 \mathrm{~dB}$. Under the same conditions, for DQSPK modulation, achieved result was $-10 \mathrm{~dB}$. From the graph shown in the figure 10 (OOK modulation) can be read that for 50 $\mathrm{GHz}$ we obtained $-15 \mathrm{~dB}$. In all cases, the use of a filter with a bandwidth of $60 \mathrm{GHz}$, was resulting in the occurrence of excessive crosstalk. Only the filter bandwidth below $40 \mathrm{GHz}$

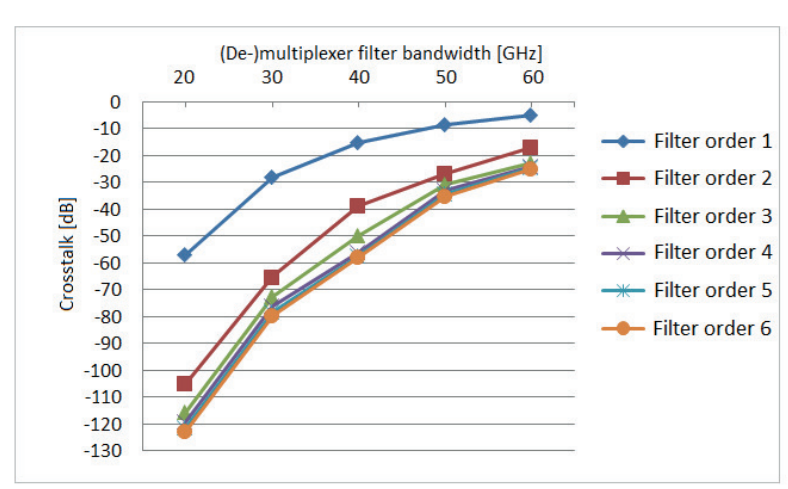

Fig. 7. Crosstalk values versus demultiplexer and multiplexer filter bandwidth for DQPSK modulation, $10 \mathrm{~Gb} / \mathrm{s}$ bit rate and different gaussian filter order. 


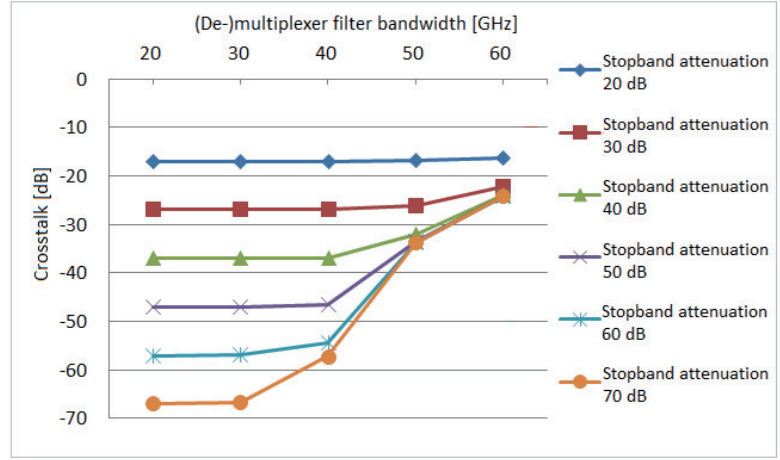

Fig. 8. Crosstalk values versus demultiplexer and multiplexer filter bandwidth for DQPSK modulation, $10 \mathrm{~Gb} / \mathrm{s}$ bit rate and different stopband attenuation of rectangular filter characteristics.

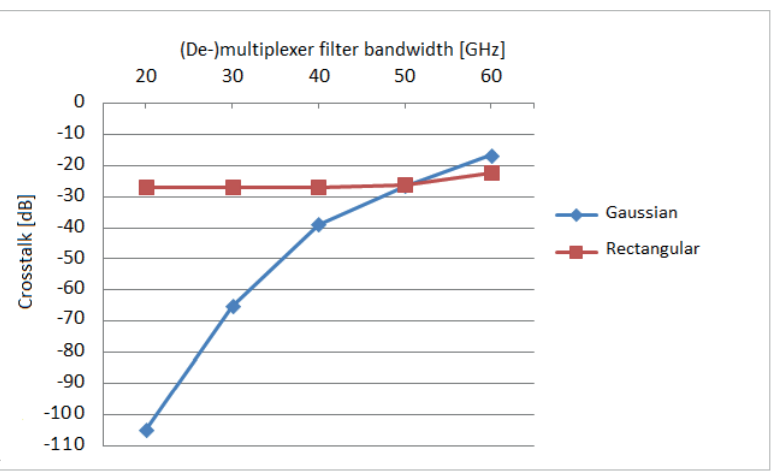

Fig. 9. Crosstalk values versus demultiplexer and multiplexer filter bandwidth for DQPSK modulation, $10 \mathrm{~Gb} / \mathrm{s}$ bit rate and different types of filters: second order gaussian filter and rectangular filter with stopband attenuation $30 \mathrm{~dB}$.

signal transmission allowed rates of $100 \mathrm{Gbit} / \mathrm{s}$. This research proved that for symmetric transmission the best results were obtained for OOK modulation. The simulation and analysis of the results showed that in most cases DQPSK modulation was better than DPSK modulation in terms of crosstalk occurring during the transmission of signals in WDM networks. This is mainly due to the fact that at the same bit rate DQPSK modulation has two times narrower bandwidth. Narrow-band filtering allowed crosstalk to be at the acceptable level $(-20$

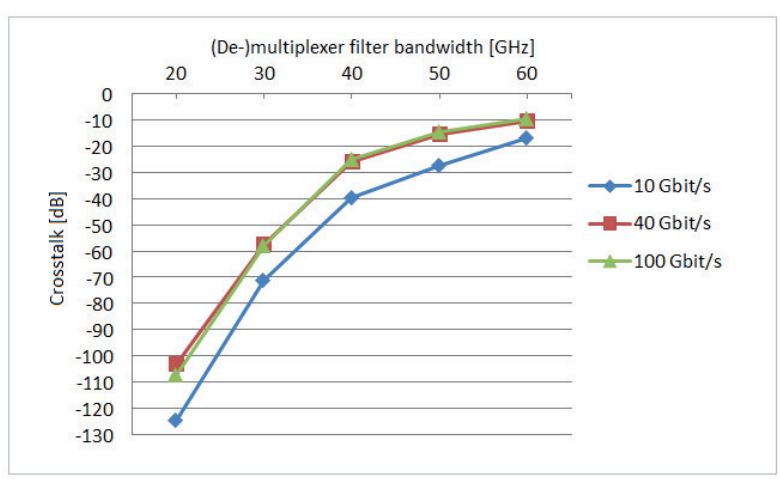

Fig. 10. Crosstalk values versus demultiplexer and multiplexer filter bandwidth for OOK modulation, second order gaussian filter and different bit rates.

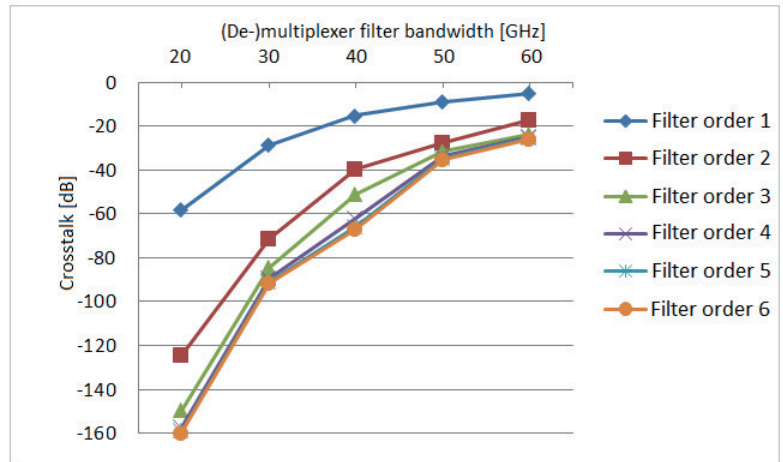

Fig. 11. Crosstalk values versus demultiplexer and multiplexer filter bandwidth for OOK modulation, $10 \mathrm{~Gb} / \mathrm{s}$ bit rate and different gaussian filter order

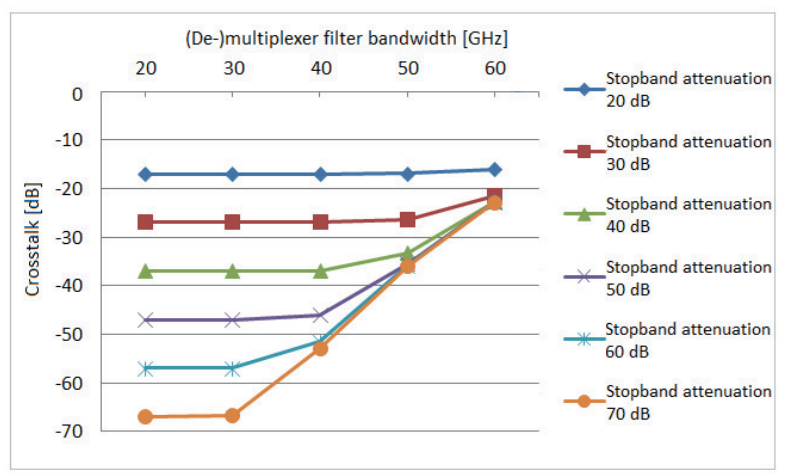

Fig. 12. Crosstalk values versus demultiplexer and multiplexer filter bandwidth for OOK modulation, $10 \mathrm{~Gb} / \mathrm{s}$ bit rate and different stopband attenuation of rectangular filter characteristics.

dB) [8]. Simulation has also shown that the maintenance of the acceptable transmission quality for filters with a width greater than $40 \mathrm{GHz}$ requires employment of $100 \mathrm{GHz}$ channel spacing. For rectangular filter it was shown that stopband attenuation might play significant role in obtaining acceptable crosstalk as well. The best results were achieved for stopband attenuation equal to $70 \mathrm{~dB}$. With the decrease of stopband attenuation the increase of crosstalk level was observed. It means that the highest level of crosstalk was obtained in

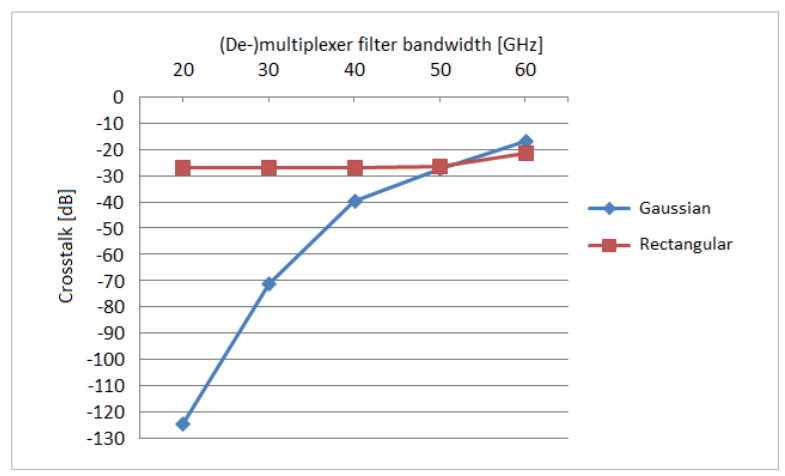

Fig. 13. Crosstalk values versus demultiplexer and multiplexer filter bandwidth for OOK modulation, $10 \mathrm{~Gb} / \mathrm{s}$ bit rate and different types of filters: second order gaussian filter and rectangular filter with stopband attenuation $30 \mathrm{~dB}$. 


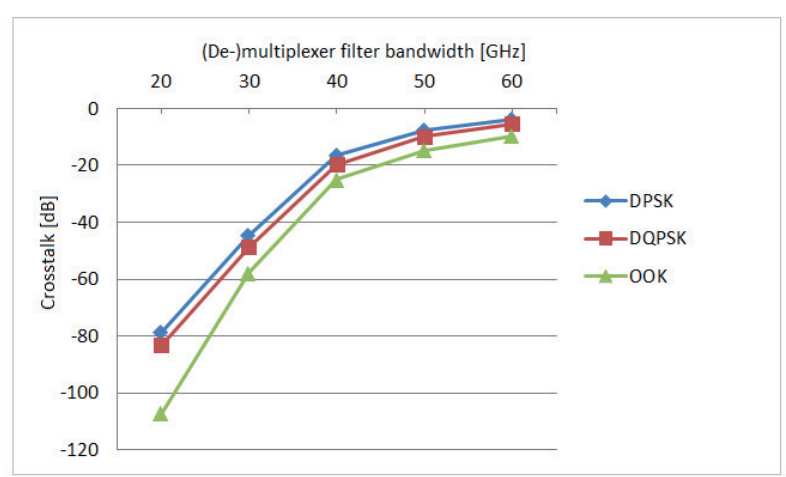

Fig. 14. Crosstalk values versus demultiplexer and multiplexer filter bandwidth for $100 \mathrm{~Gb} / \mathrm{s}$ bit rate, second order gaussian filter and different types of modulation.

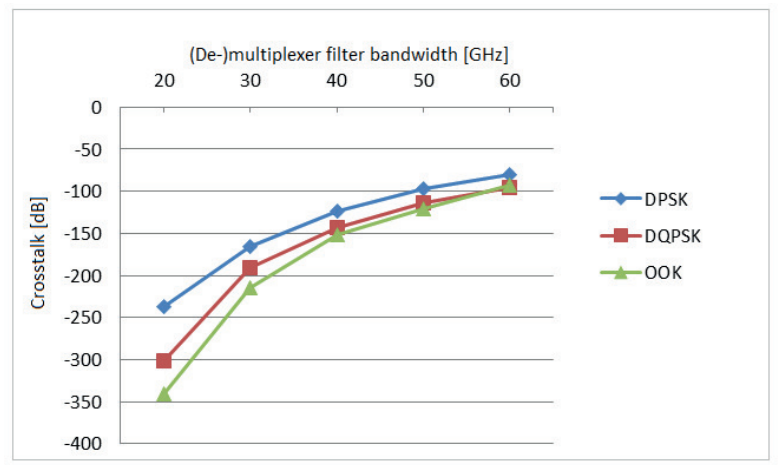

Fig. 15. Crosstalk values versus demultiplexer and multiplexer filter bandwidth for $100 \mathrm{~Gb} / \mathrm{s}$ transmission, second order gaussian filter and different types of modulation $(100 \mathrm{GHz}$ channel spacing).

case of $20 \mathrm{~dB}$ stopband attenuation for all modulations. For gaussian filters of $4^{\text {th }}-6^{\text {th }}$ order there were achieved the best results. In case of filters $1^{\text {st }}-3^{\text {rd }}$ order level of crosstalk was excessively high. This is because the lower order transfer functions have much gentler slopes, which contributes to the higher power signal outside the desired band of gaussian filter. In case of higher order filters slopes are steeper. This is the reason why signal power is better attenuated between two channels. Therefore, each channel is properly separated

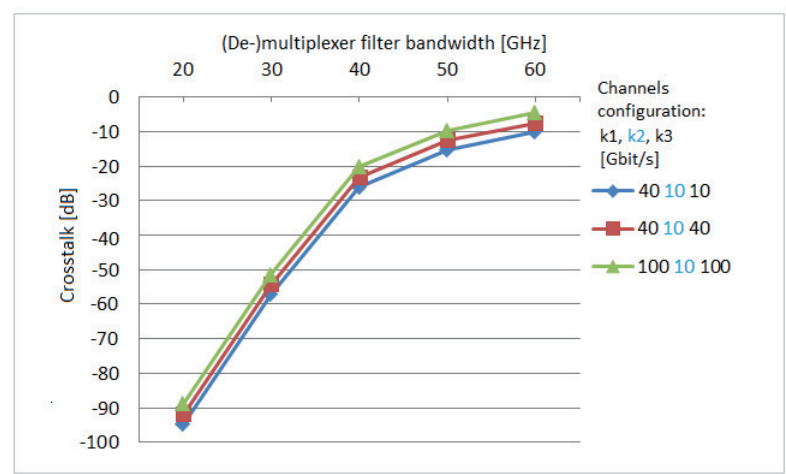

Fig. 16. Crosstalk values versus demultiplexer and multiplexer filter bandwidth for DPSK modulation, second order gaussian filter and different configuration of bit rates in adjacent channels.

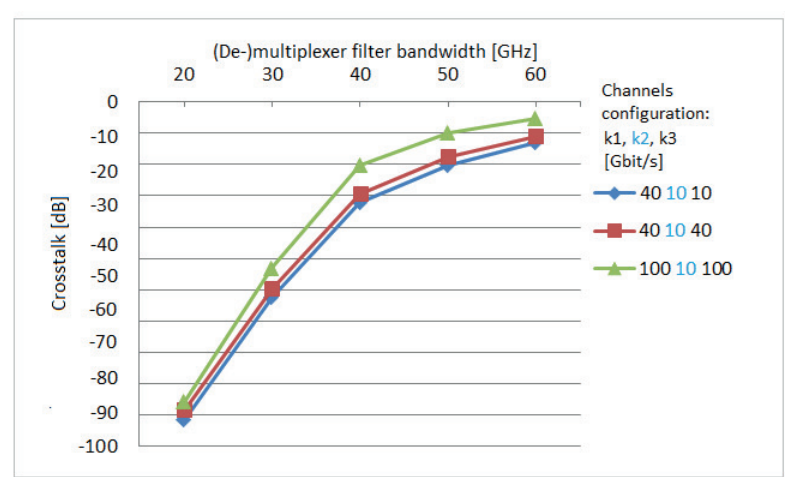

Fig. 17. Crosstalk values versus demultiplexer and multiplexer filter bandwidth for DQPSK modulation, second order gaussian filter and different configuration of bit rates in adjacent channels.

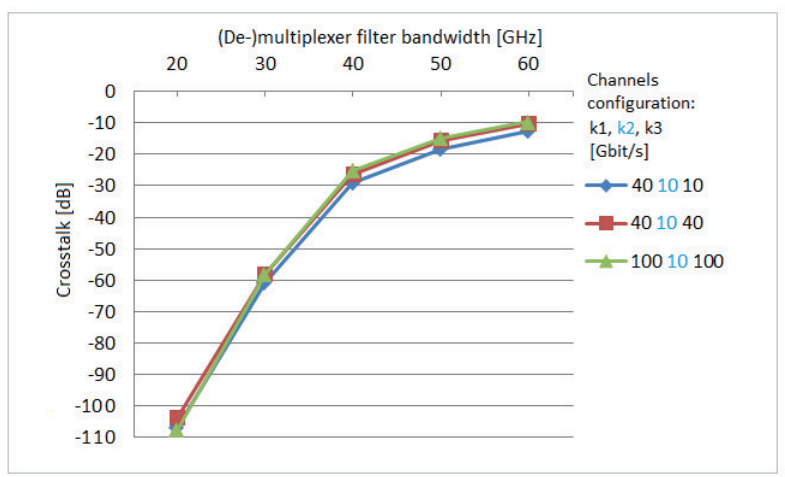

Fig. 18. Crosstalk values versus demultiplexer and multiplexer filter bandwidth for OOK modulation, second order gaussian filter and different configuration of bit rates in adjacent channels.

from the others. Comparison between gaussian and rectangular filter for DPSK modulation was shown in figure 5. There were compared: rectangular filter with stopband attenuation of $30 \mathrm{~dB}$ and $2^{\text {nd }}$ order gaussian filter. In this case a better solution was the rectangular filter, for which obtained level of crosstalk was lower than in case of a gaussian filter. For example for $50 \mathrm{GHz}$ bandwidth of gaussian filter, crosstalk was about $-23 \mathrm{~dB}$ and for the rectangular was around $15 \mathrm{~dB}$ lower.

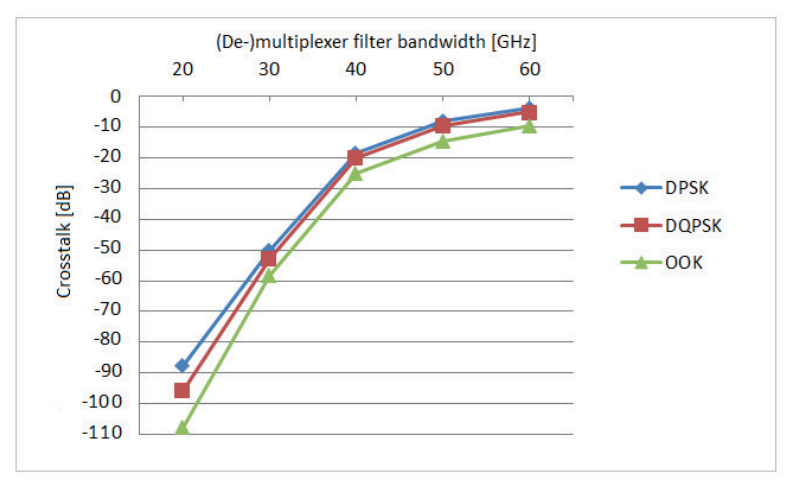

Fig. 19. Crosstalk values versus demultiplexer and multiplexer filter bandwidth for different types of modulation, second order gaussian filter and bit rate $10 \mathrm{~Gb} / \mathrm{s}$ in tested channel and $100 \mathrm{~Gb} / \mathrm{s}$ for adjacent channels. 
TABLE I

Bit RATES CONFIGURATION FOR THE ASYMMETRIC TRANSMISSION

\begin{tabular}{cccc}
\hline \hline $\begin{array}{c}\text { Number of } \\
\text { channel }\end{array}$ & Channel 1 & Channel 2 & Channel 3 \\
\hline \multirow{3}{*}{ Bit Rate } & $40 \mathrm{~Gb} / \mathrm{s}$ & $10 \mathrm{~Gb} / \mathrm{s}$ & $10 \mathrm{~Gb} / \mathrm{s}$ \\
& $40 \mathrm{~Gb} / \mathrm{s}$ & $10 \mathrm{~Gb} / \mathrm{s}$ & $100 \mathrm{~Gb} / \mathrm{s}$ \\
& $100 \mathrm{~Gb} / \mathrm{s}$ & $10 \mathrm{~Gb} / \mathrm{s}$ & $100 \mathrm{~Gb} / \mathrm{s}$ \\
\hline \hline
\end{tabular}

Only for narrow-band filtering the situation was different and rectangular filter had worse results. For DQPSK modulation plots look slightly different (Fig. 9). The experimental results were much better in case of use of gaussian filter than the rectangular. Only filter bandwidth of $60 \mathrm{GHz}$ for rectangular characteristic of filter turned out to be better. Below this value, the level of crosstalk for the $2^{\text {nd }}$ order gaussian filter decreased significantly and at $20 \mathrm{GHz}$ band of the filter is less than $-100 \mathrm{~dB}$. For OOK modulation the best results were achieved for gaussian filter as well. Figs. 16-19 show the results for case 2 (asymmetric transmission). In the channel, which was considered for calculating crosstalk, the bit rate was $10 \mathrm{~Gb} / \mathrm{s}$, while in the adjacent channels signals were transmitted with $40 \mathrm{~Gb} / \mathrm{s}$ or $100 \mathrm{~Gb} / \mathrm{s}$. There was also used $2^{\text {nd }}$ order gaussian filter in simulation setup. Tab. I presents configuration of channels used for asymmetric transmission. Simulations were performed for the WDM system shown in Fig. 1.

The dependency presented in the Figs. 13-16 indicates an increase in level of crosstalk with the transmission at higher bit rate in adjacent channels. As expected the lowest crosstalk was obtained for bit rate of $40 \mathrm{Gbit} / \mathrm{s}$ in channel 1 and $10 \mathrm{Gbit} / \mathrm{s}$ in channel 3 and the transmission of $100 \mathrm{Gbit} / \mathrm{s}$ on both adjacent channels resulted in the occurrence of the highest crosstalk. For DQPSK modulation level of crosstalk was slightly lower than for DPSK. For $50 \mathrm{GHz}$ (de)multiplexer filter bandwidth and transmission in channels 1-40 Gbit/s, 2-10 Gbit/s and 3-40 Gbit/s, the level of crosstalk was equal to $-18 \mathrm{~dB}$. This value was obtained for DPSK modulation with (de)multiplexer bandwidth of $45 \mathrm{GHz}$. It means that receiving the same results for DPSK modulation requires narrower bandwidth of filter. In case of OOK modulation, the obtained results show improvement over previous tested modulations. Figure 19 illustrates comparison of the level of crosstalk for asymmetric transmission for the three tested modulations. In this situation for $40 \mathrm{GHz}$ of the filter bandwidth of (de)multiplexer, bit rate $10 \mathrm{Gbit} / \mathrm{s}$, transmission of $100 \mathrm{Gbit} / \mathrm{s}$ in adjacent channels and OOK modulation data transfer was possible - the level of crosstalk was around $-25 \mathrm{~dB}$. Under the same conditions for DPSK and DQPSK modulation, crosstalk was $-20 \mathrm{~dB}$. So for asymmetric transmission level of crosstalk is the lowest for OOK modulation. It can also be concluded that acceptable crosstalk is achieved for transmission $100 \mathrm{~Gb} / \mathrm{s}$ in adjacent channels only with the narrow-band filtering $(30 \mathrm{GHz}$ filter bandwidth). For lower bit rates bandwidth of $40 \mathrm{GHz}-50 \mathrm{GHz}$ is sufficient for all modulations.

\section{CONCLUSION}

The obtained results indicate that for asymmetric and symmetric transmission the best results were obtained for OOK modulation. The simulation and analysis of the results showed that in most cases DQPSK modulation is better than DPSK modulation in terms of crosstalk occurring during the transmission of signals in WDM networks. It was also proved that for DPSK modulation there can be achieved better results for rectangular filter. In case of DQPSK and OOK modulation gaussian filter turned out to be better choice. Another drawn conclusion is that narrow-band filtering plays significant role in obtaining allowable level of crosstalk. Improvement of results may also be achieved by increasing the channel spacing from $50 \mathrm{GHz}$ to $100 \mathrm{GHz}$.

\section{REFERENCES}

[1] O. Gerstel, R. Cassata, and L. Paraschis, "Operational Solutions for an Open DWDM layer," in Conference on Optical Fiber Communication/National Fiber Optic Engineers Conference, NthF1, San Diego, USA, 2009.

[2] S. Melle, G. Bennett, and C. Liou, "Alien Wavelength Transport," in An Operational and Economic Analysis Conference on Optical Fiber Communication/National Fiber Optic Engineers Conference, NthF2, San Diego, USA, 2009.

[3] A. Hodzic and M. Winter, "Optimized Filtering for $40-\mathrm{Gb} / \mathrm{s} / \mathrm{Ch}-\mathrm{Based}$ DWDM Transmission Systems Over Standard Single-Mode Fiber," Photonics Technology Letters, vol. 7, pp. 1645-1647, 2011.

[4] S. Suzuki and Y. Kokubun, "Design Rule of Wavelength Filter Bandwidth and Pulsewidth for Ultimate Spectral Efficiency Limited by Crosstalk in DWDM Systems," Photonics Technology Letters, vol. 11, pp. 1002-1004, 2003.

[5] K. Perlicki, "Impact of an alien wavelength on wavelength division multiplexing transmission quality," Photonics Letters of Poland, vol. 3, pp. $118-120,2012$

[6] A. Kowalczyk, "Wpływ parametrów elementw filtracyjnych na jakość pracy systemów WDM," in Bachelor of science thesis, Institute of Telecommuniacations, Warsaw University of Technology, Warsaw, Poland, 2013.

[7] ITU-T G.694.1, "Spectral grids for WDM applications," in DWDM frequency grid, Geneva, Switzerland, 2/2012.

[8] G. P. Agrawal, Fiber-Optic Communication Systems. New York, USA: A John Wiley \& Sons, Inc., 2002. 